\title{
Investigation of aerosol dispersion in fibrous filters
}

\author{
${ }^{1}$ Anna Jackiewicz, 1, ${ }^{2}$ Anna Bałazy, ${ }^{1}$ Albert Podgórski \\ ${ }^{1}$ Faculty of Chemical and Process Engineering, Warsaw University of Technology, ul. Waryńskiego 1, 00-645 Warszawa, \\ Poland, e-mail: jackiewicz@ichip.pw.edu.pl \\ ${ }^{2}$ Cummins Filtration, Inc., 1801 U.S. Hwy. 51/138, Stoughton, WI 53589-0428, USA
}

Fractional penetration through four fibrous filters was determined experimentally at various air velocities. To describe the data obtained for multilayer sets of filters composed of up to nine layers of the same filter, the axial dispersion model was applied. This model predicts a non-exponential decrease of aerosol penetration with a filter thickness increase. The dispersion model contains two parameters, $\lambda$ and $D_{x}$, which were evaluated on the basis of the experimental data. For the first time ever the axial dispersion coefficients, $D_{x}$, in fibrous filters were determined.

Keywords: aerosol dispersion, fibrous filters, filtration.

\section{INTRODUCTION}

Fibrous filters are very efficient for the removal of sub- and micrometer particles. The advantages of such kind of filters are their high efficiency and, at the same time, relatively low pressure drop. The filter efficiency depends on its structural parameters, such as porosity, fiber diameter, thickness, and on the degree of its inhomogeneity. Aerosol filtration in fibrous filters is usually considered as a depth filtration, which means that the particles are deposited within the entire volume of the filter. According to the classical theory of depth filtration the aerosol penetration, $P$, is expected to be an exponentially decreasing function of the filter thickness, $L$. Thus, $P=\exp (-\lambda L)$, wherein the filter coefficient, $\lambda$, is related to the single fiber efficiency, $E$, the filter porosity, $\varepsilon$, and the fiber diameter, $d_{f}$ according to the formula: $\lambda=4 E(1-\varepsilon) / \pi \varepsilon d_{f}$. To optimize the filtration process, the multilayer sets of fibrous filters are recommended ${ }^{1}$. In such a case, the penetration should be - according to the classical theory of depth filtration - a product of the particle penetrations through each layer. Such an approach does not take into consideration possible mass dispersion in an inhomogeneous filter that would be caused by preferential transport of aerosol particles through the zones of a higher local permeability. This phenomenon of mass dispersion in fibrous materials affects in turn their efficiency. The mass dispersion in porous media was a subject of many theoretical and experimental papers $^{2-4}$. Unfortunately, most of the experimental data available in the literature concern granular beds, while there is a lack of such information for the fibrous filters, which are far more porous than the typical beds. The aim of this study was to examine if the mass dispersion may be a significant phenomenon in the fibrous filters and to determine the values of the coefficients of axial dispersion.

\section{MATERIALS AND METHODS}

\section{Characteristics of the filters' structures}

Four fibrous polypropylene filters made by us, utilizing the melt-blown technique, were used to perform the experiments. Their structures were fully characterized and the results are collected in Table 1. The following parameters of the filters' media were determined: mean fiber diameter, $d_{f}$, filter thickness, $L$, porosity, $\varepsilon$ and surface density, $q_{s}$. The degree of the fibers' polydispersity can be characterized by the coefficient of variation, $C V$, defined as the ratio of the standard deviation of fibers' size distribution, $S D$, to the mean fiber diameter. Fig. 1 depicts the fibers' size distributions and the images of all the tested filters taken under a microscope.

Amongst the tested filters, the filter \#1 was the thinnest one and had the lowest mean fiber diameter, while the filter \#4 was the thickest one and its mean fiber diameter was the biggest. The filter \#1 was the least densely packed and its surface density was significantly lower than the one for other filters. The most homogeneous structures of all the tested filters had filters $\# 1$ and \#4. The other fibrous filters were more polydisperse with $C V$ ranging between 0.4 and 0.6.

\section{DETERMINATION OF THE FILTER EFFICIENCY}

\section{Experimental setup}

Fractional penetration of solid aerosol particles with diameters $0.2-10 \mu \mathrm{m}$ through four fibrous filters was determined using the modular filter test system (model MFP-2000, Palas GmbH, Germany), which is shown schematically in Fig. 2. The experiments were carried out for several air velocities in the range $u=0.08-0.2 \mathrm{~m} / \mathrm{s}$.

The compressed air was thoroughly purified in filter 3 before supplying it to a rotating brush aerosol generator 4 (model RBG 1000, Palas GmbH) and to the mass flow controller 6 . In neutralizer 8 the generated aerosol was mixed with the bipolarly ionized gas and additionally diluted by clean air. The neutralized aerosol was directed to an exposure chamber 10, where the tested filer 12 was kept. A number size distribution of aerosol particles was measured using an optical particle counter 15 (model PCS 2010, Palas GmbH). A pump 17 (model ASP 2000, Palas GmbH) served to suck a steady volume of the test aerosol.

\section{Fractional efficiency - multilayer sets}

The initial collection efficiencies of aerosol particles through a different number of layers of two filters are shown in Fig. 3. The experiments with filter \#3 were carried out at the air velocity of $0.08 \mathrm{~m} / \mathrm{s}$, while the filter \#2 was tested at $u=0.2 \mathrm{~m} / \mathrm{s}$. In both cases the experiments were performed for up to nine layers of the same filter. The results shown in Fig. 3 indicate that addition of a filter layer decreases the number of particles that penetrate through the filter, and such a decrease is particularly seen for larger particles. 
Table 1. The characteristics of the investigated filters

\begin{tabular}{|l|c|c|c|c|c|}
\hline Filter number & $\begin{array}{c}\text { Mean fiber } \\
\text { diameter, } \\
d_{f}[\mu \mathrm{m}]\end{array}$ & $\begin{array}{c}\text { Filter thickness, } \\
L[\mathrm{~mm}]\end{array}$ & $\begin{array}{c}\text { Filter porosity, } \\
\varepsilon[\%]\end{array}$ & $\begin{array}{c}\text { Coefficient of variation } \\
C V[-]\end{array}$ & $\begin{array}{c}\text { Filter surface density, } \\
q_{s}\left[\mathrm{~g} / \mathrm{m}^{2}\right]\end{array}$ \\
\hline 1 & $11.9 \pm 3.8$ & 0.77 & 93.2 & 0.32 & 47 \\
\hline 2 & $15.0 \pm 6.2$ & 1.28 & 84.4 & 0.41 & 182 \\
\hline 3 & $12.5 \pm 7.6$ & 1.14 & 82.2 & 0.61 & 184 \\
\hline 4 & $25.4 \pm 8.4$ & 1.37 & 83.6 & 0.33 & 205 \\
\hline
\end{tabular}
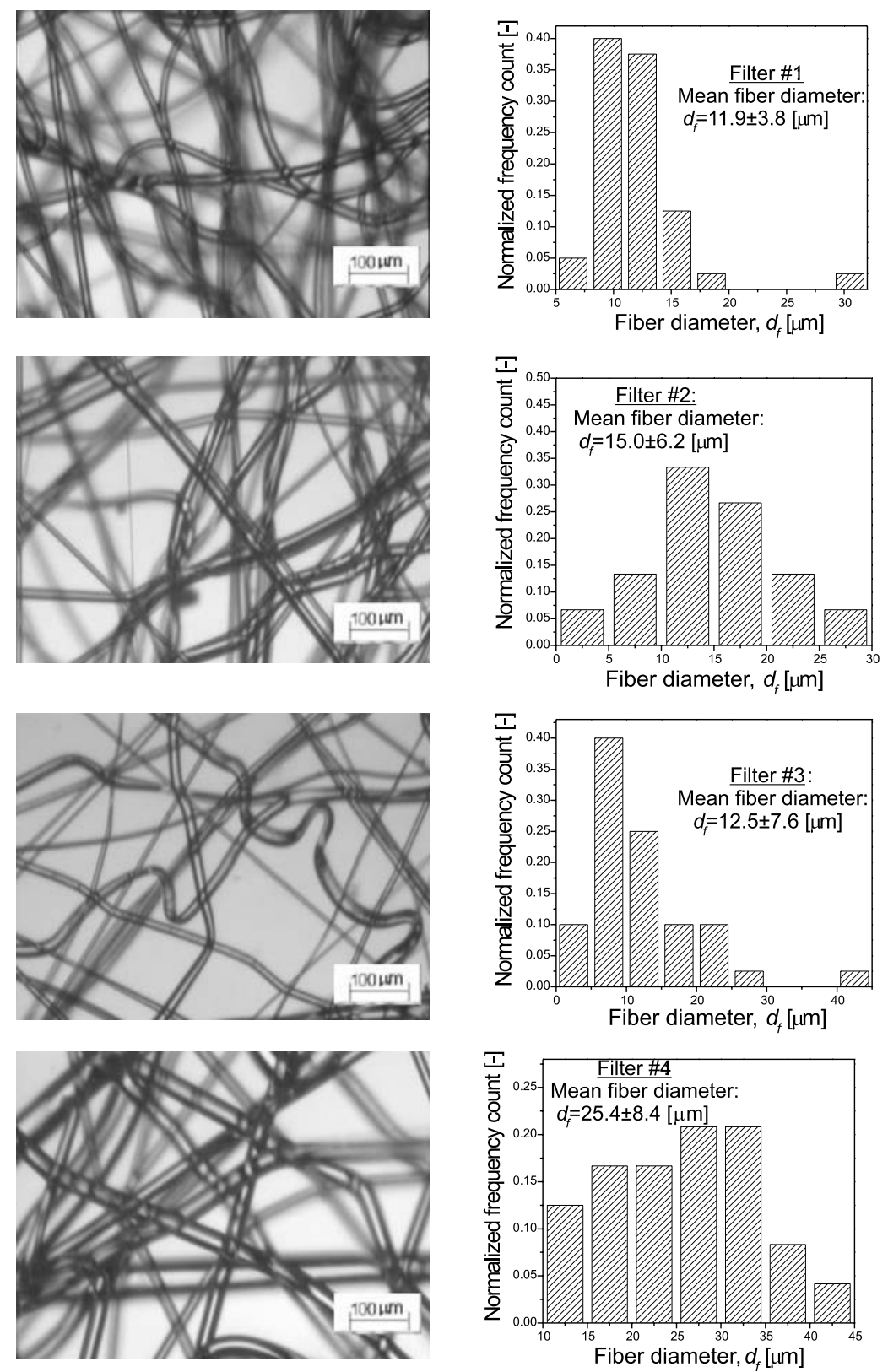

Figure 1. Microscopic images and fibers' size distributions of all the tested filters

\section{MODEL OF AXIAL DISPERSION IN FIBROUS FIL- TERS}

To describe the data obtained for the multilayer sets of fibrous filters composed of up to nine layers of the same filter, the axial dispersion model was applied. This model predicts a nonexponential decrease of aerosol penetration with the filter thickness, $L$. It is based on the macrotransport equation for aerosol filtration in fibrous filters:

$u \frac{\partial c}{\partial x}-\varepsilon D_{x} \frac{\partial^{2} c}{\partial x^{2}}+\lambda u c=0$

Eq. (1) can be integrated with Danckwerts' boundary condi- inlet: $u c_{0}=u c_{\mid x=0^{+}}-\varepsilon D_{x}\left(\frac{\partial c}{\partial x}\right)_{\mid x=0^{+}}$, outlet: $D_{x}\left(\frac{\partial c}{\partial x}\right)_{\mid x=L}=0$.

In the above equations $D_{x}$ is the coefficient of axial dispersion, $c$ means a momentary, cross-section-averaged aerosol concentration, and $x$ is the distance measured from the filter inlet. The solution of the standard axial dispersion model for aerosol filtration in fibrous filters with Danckwerts' boundary conditions has the following form ${ }^{5}$ : tions: 


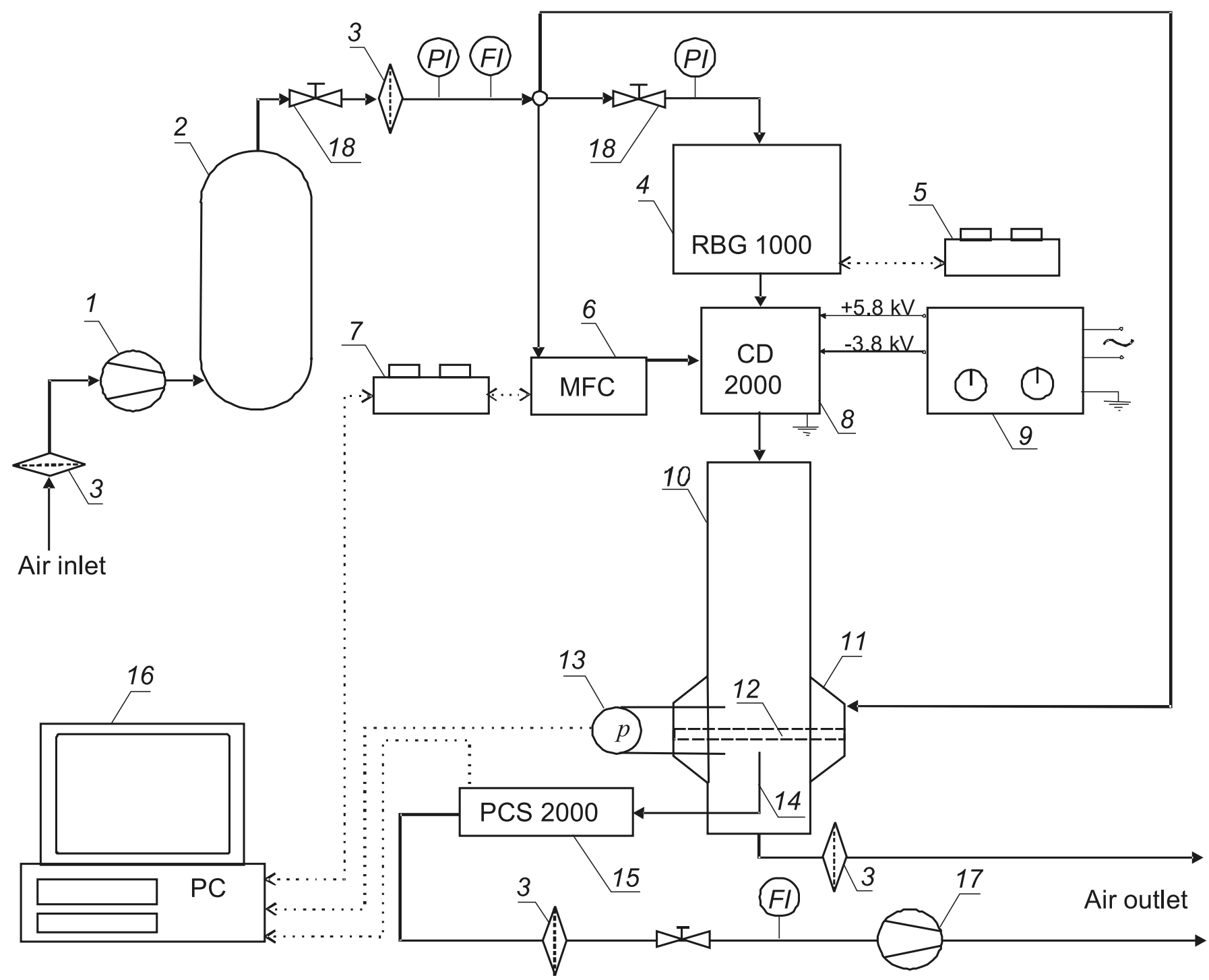

Figure 2. A schematic diagram of the experimental setup: 1 - compressor, 2 - pressure vessel, 3 - absolute filter, 4 - generator of solid aerosol particles, 5 - operating panel of the aerosol generator, 6 - mass flow controller, 7 - operating panel of the mass flow controller, 8 - corona discharge unit, 9 - double high voltage supplier, 10 - exposure chamber, 11 - pneumatic filter holder, 12 - tested filter, 13 - differential pressure transducer, 14 - sampling nozzle, 15 - optical particle counter, 16 - computer, 17 - suction pump, 18 - reduction valve

a)

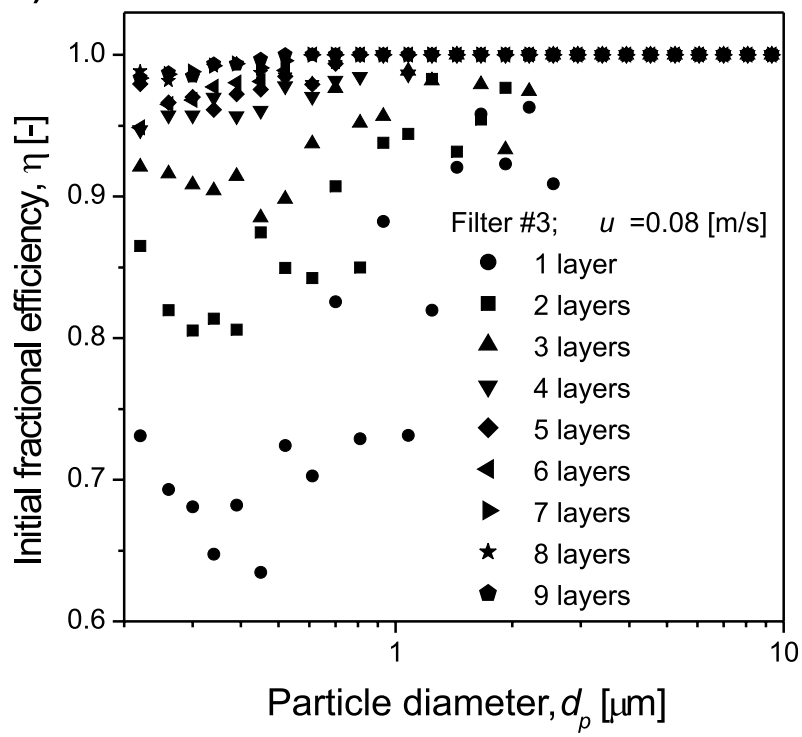

b)

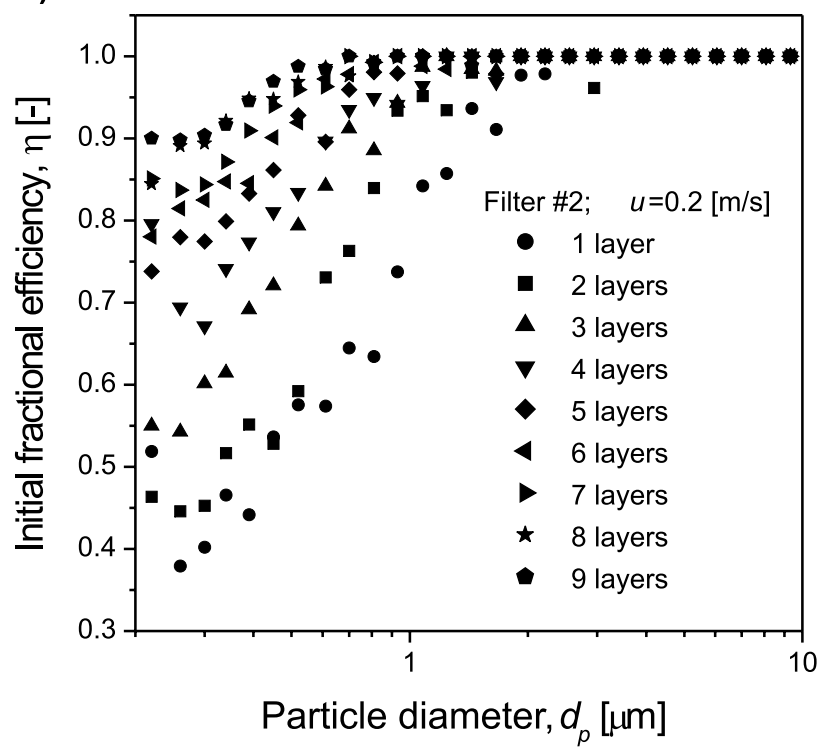

Figure 3. The influence of the filter thickness on the initial fractional efficiency for the multilayer sets of the filter \#2 and the filter \#3 composed of up to nine layers of the same filter 


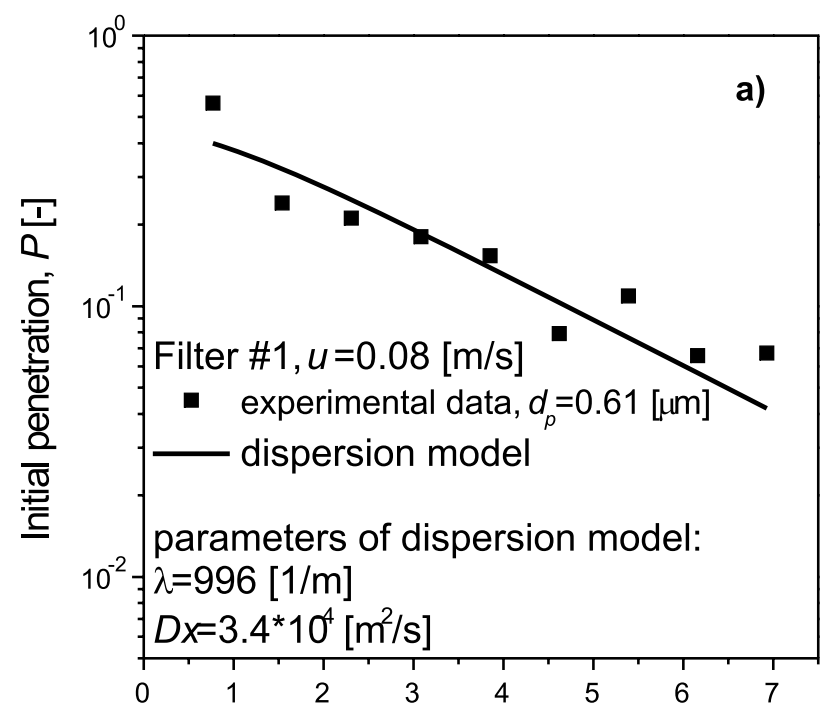

Filter thickness, $L$ [mm]

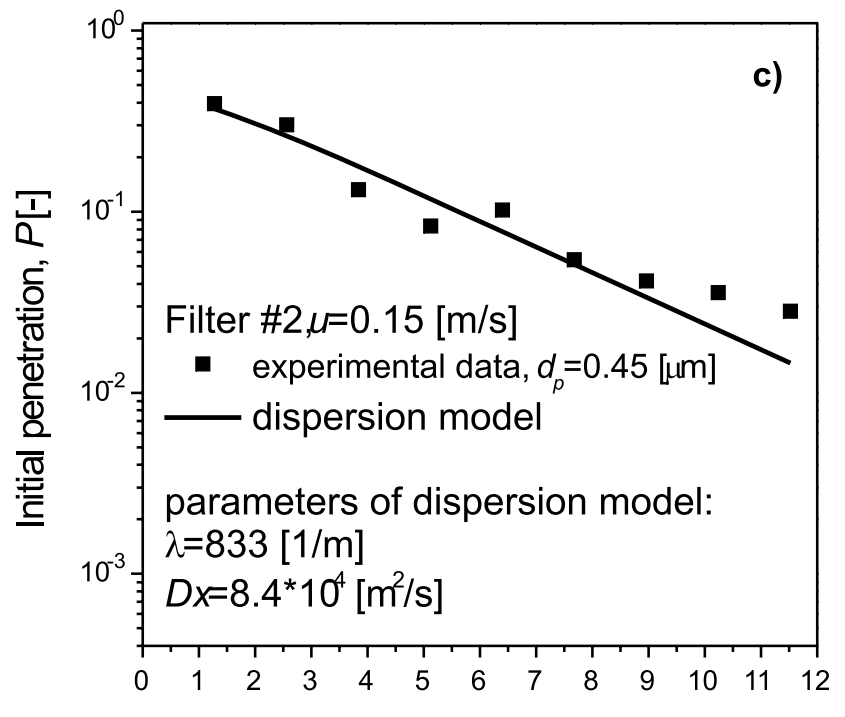

Filter thickness, $L[\mathrm{~mm}]$

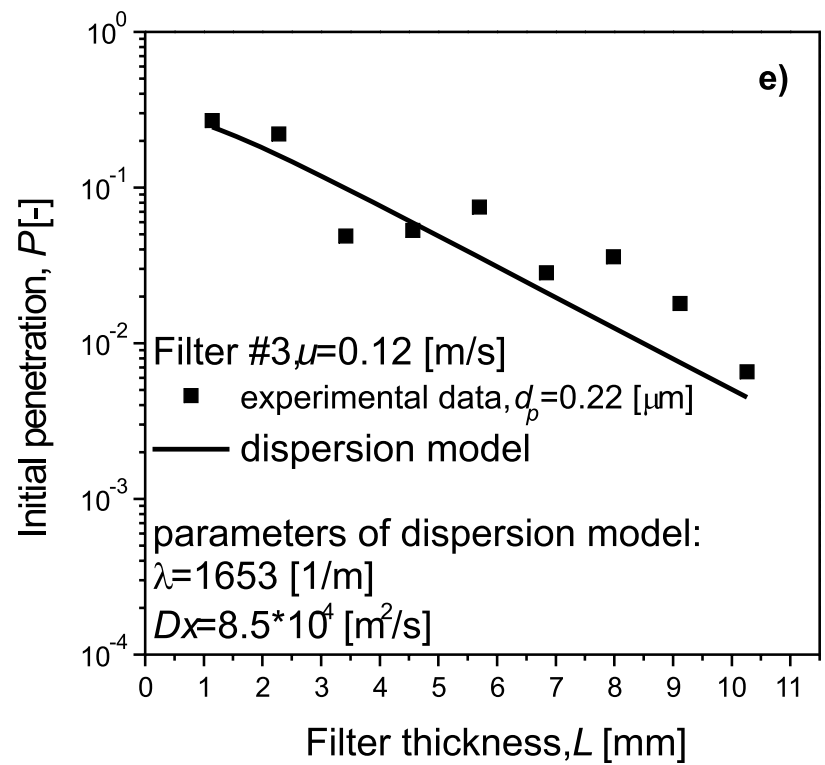

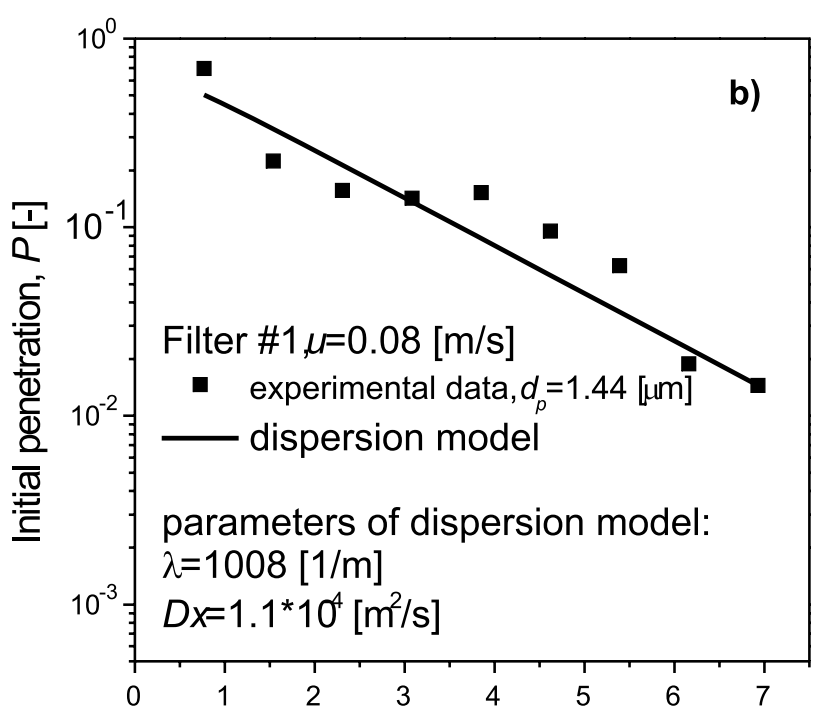

Filter thickness, $L$ [mm]

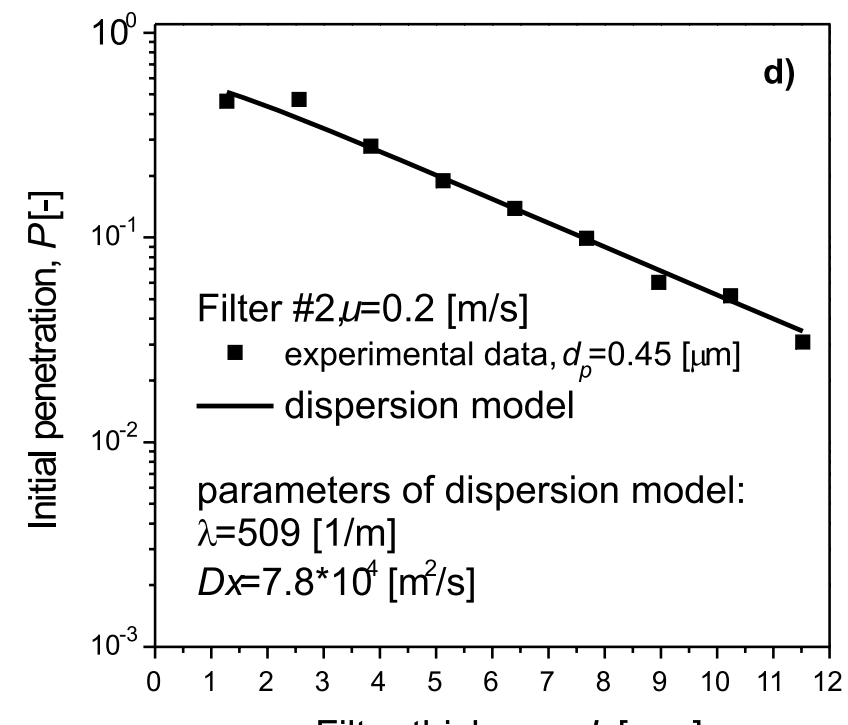

Filter thickness, $L$ [mm]

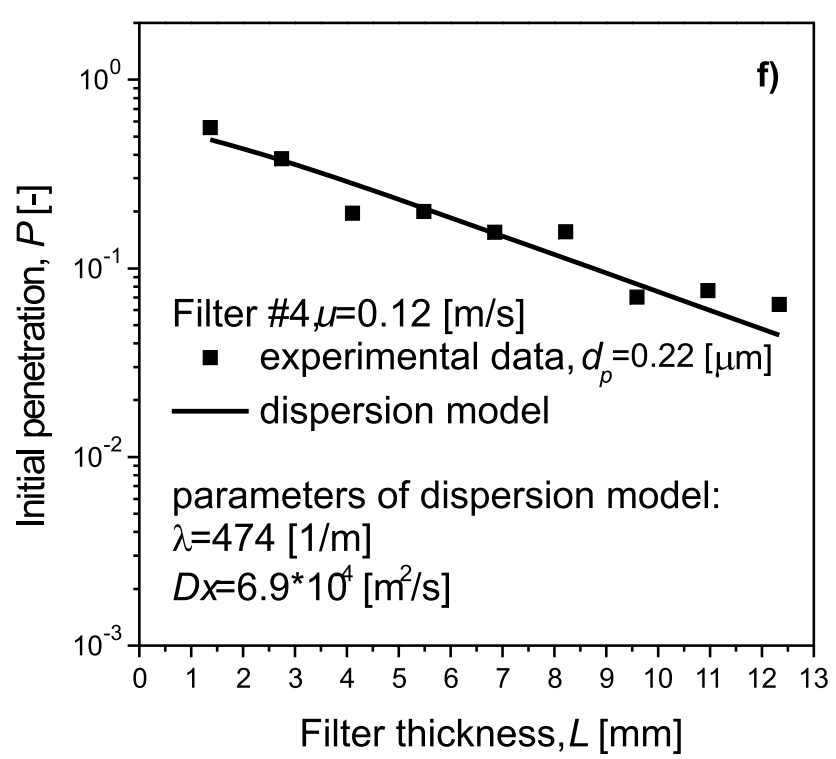

Figure 4. Application of the dispersion model to fit the experimental data of particles' penetration through the nine layers of the filters \#1-\#4 for several air velocities and several particle diameters 


$$
\begin{aligned}
& \frac{1}{P}=\frac{\left(1+\sqrt{1+\frac{4 d_{f} \lambda}{\mathrm{Bo}}}\right)^{2}}{4 \sqrt{1+\frac{4 d_{f} \lambda}{\mathrm{Bo}}}} \exp \left[-\left(1-\sqrt{1+\frac{4 d_{f} \lambda}{\mathrm{Bo}}}\right) \frac{L \mathrm{Bo}}{2 d_{f}}\right]+ \\
& +\frac{\left(1-\sqrt{1+\frac{4 d_{f} \lambda}{\mathrm{Bo}}}\right)^{2}}{4 \sqrt{1+\frac{4 d_{f} \lambda}{\mathrm{Bo}}}} \exp \left[-\left(1+\sqrt{1+\frac{4 d_{f} \lambda}{\mathrm{Bo}}}\right) \frac{L \mathrm{Bo}}{2 d_{f}}\right]
\end{aligned}
$$

where the Bodenstein number is defined as follows: Bo $=u d_{f} / \varepsilon D_{x}$.

\section{RESULTS AND DISCUSSION}

Having determined the experimental data of aerosol penetration as a function of the filter thickness, the coefficients of axial dispersion were calculated by fitting the data to the Eq. (3), wherein $D_{x}$ and $\lambda$ were the free parameters. A good agreement between the model described above and the experimental results was obtained in each case, see Fig. 4.

The determined values of the $\lambda$ parameter, which is proportional to the single fiber efficiency, are shown in Fig. 5 as a function of the particle diameter for various air velocities. It can be observed that the filter coefficient decreases with the increase

a)

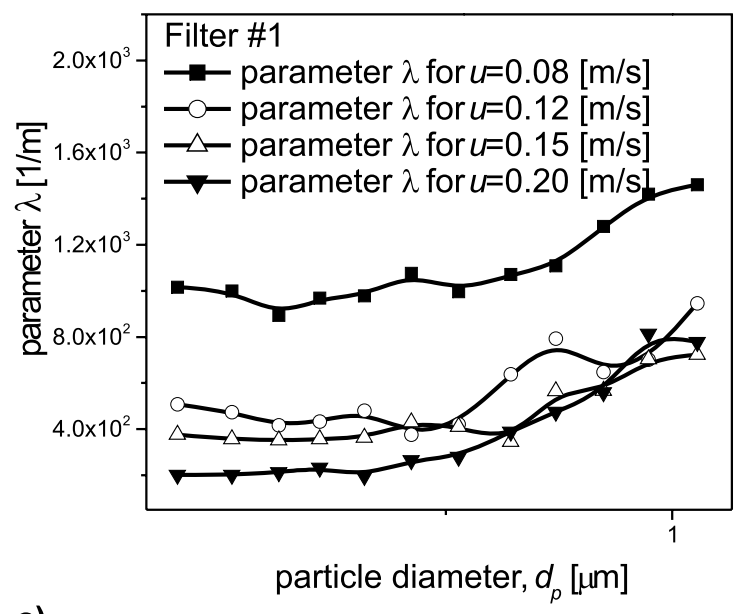

c)

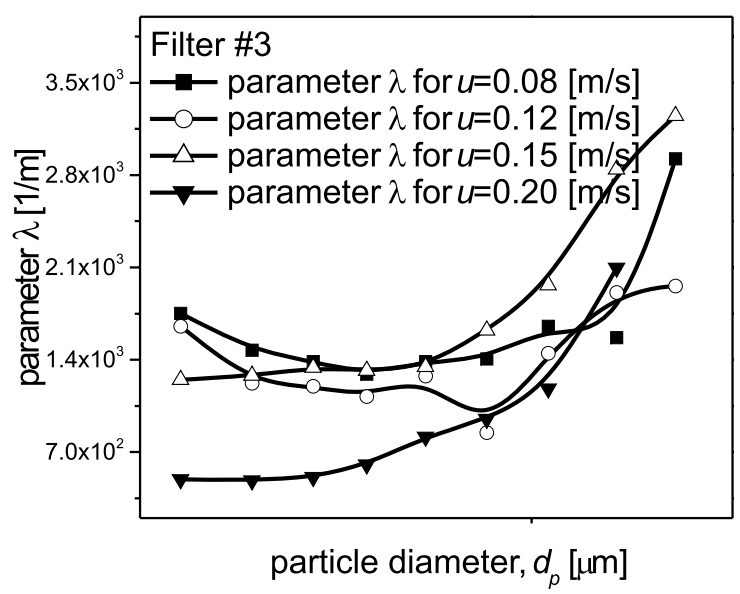

of the gas velocity when the diffusional mechanism dominates (for the particles smaller than $0.3 \mu \mathrm{m}$ ). For the larger particles, when the deterministic mechanisms of deposition predominate, an inverse relationship can be observed i.e., $\lambda$ increases with the increase of $u$. It means that for large particles the mechanism of inertial impaction is more significant than the deposition due to direct interception. It can be seen in Fig. 5 that the coefficient $\lambda$ reaches the minimum values for the particles' diameters in the range of $0.3-0.4 \mu \mathrm{m}$ (the most penetrating particle size).

Simultaneously with the determination of the $\lambda$ parameter, the values of the coefficients of axial dispersion, $D_{x}$, were calculated by fitting the experimental data of aerosol penetration in the multilayer sets of filters to Eq. (3). It was found that the coefficients of axial dispersion, $D_{x}$, are a few orders of magnitude greater than the values of the Brownian diffusion coefficients, $D$, determined for the same particle diameters. $D$ can be calculated from the Stokes-Einstein formula: $D=k_{B} T C_{c} / 3 \pi \mu_{g} d_{p}$, wherein $k_{B}$ is the Boltzmann constant, $T$ is the absolute temperature, $C_{C}$ is the Cunningham slip correction factor and $\mu_{g}$ is the gas viscosity.

It is commonly used to apply the following correlation: $D_{x} / D=A \mathrm{Pe}^{\mathrm{m}}$ to approximate the dependence of the dimensionless dispersivity, defined as the ratio of the dispersion coefficient, $D_{x}$, to the coefficient of Brownian diffusion, $D$, on the Peclet number ${ }^{\mathbf{6}}$. The Peclet number is defined as: $\mathrm{Pe}=u d_{f} / D$.

The results gained for all the tested filters at several air velocities are shown in Fig. 6. The ratio of the dispersion

b)

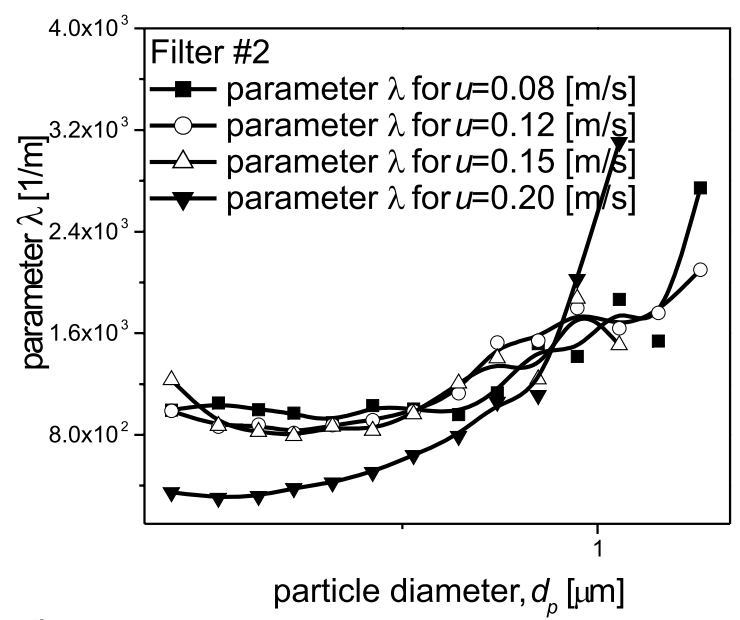

d)

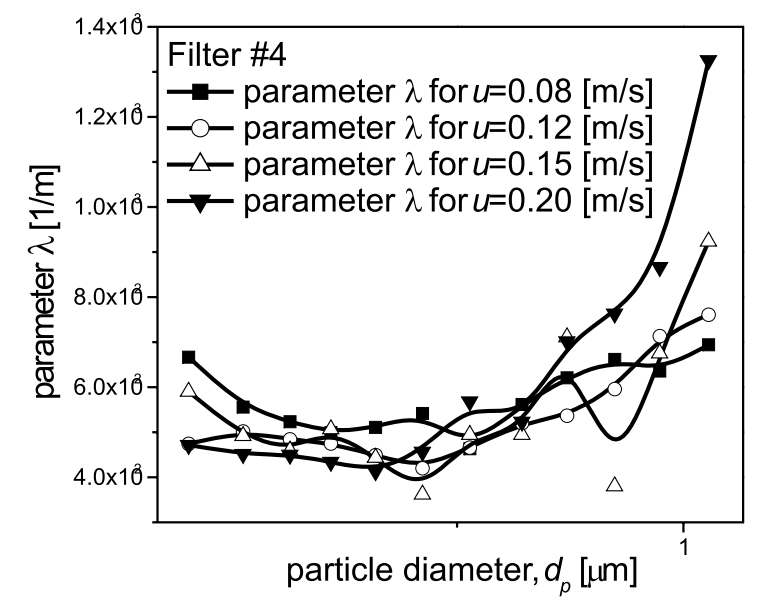

Figure 5. The values of the $\lambda$ parameter vs the particle diameter, $d_{p}$, for several air velocities 
a)

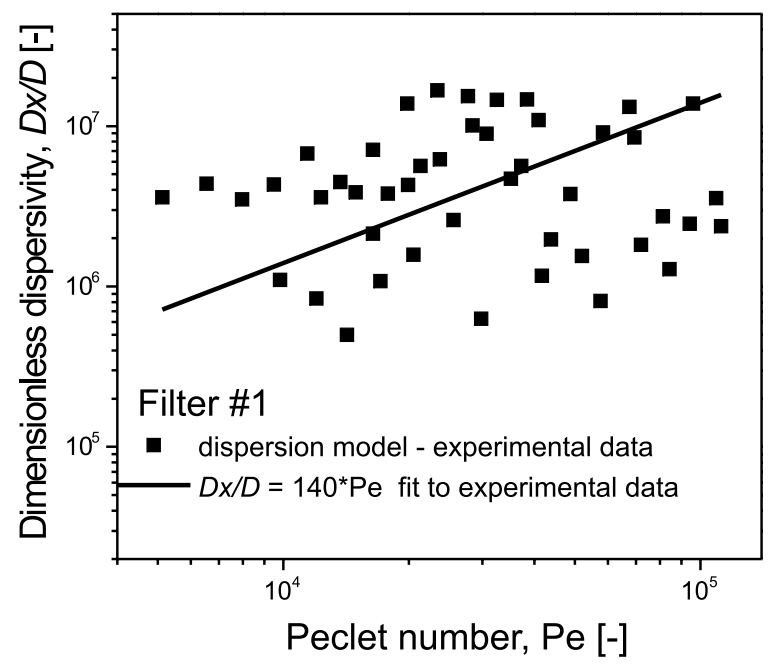

c)

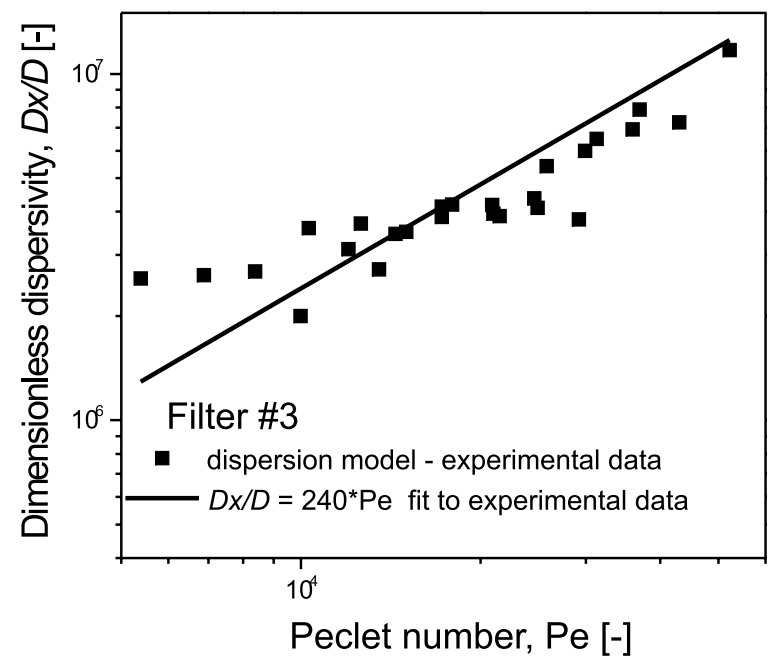

b)

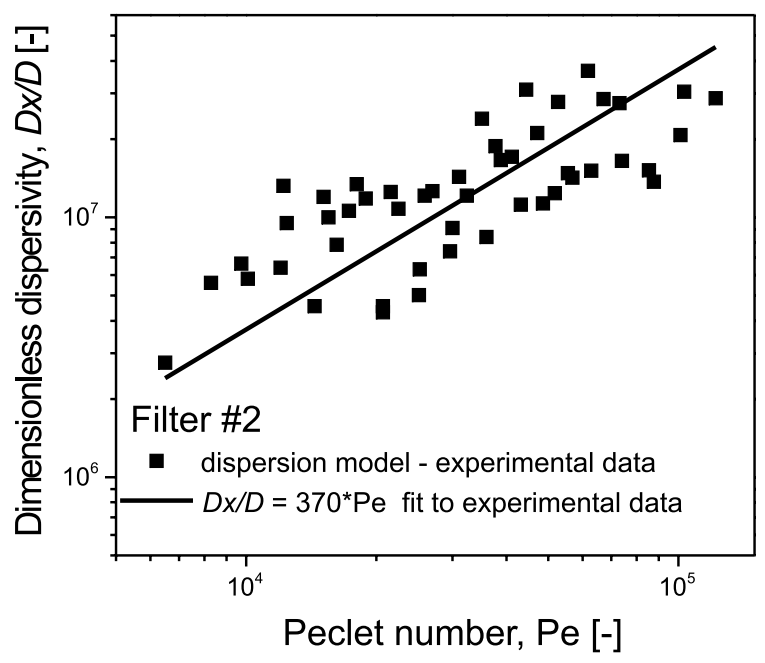

d)

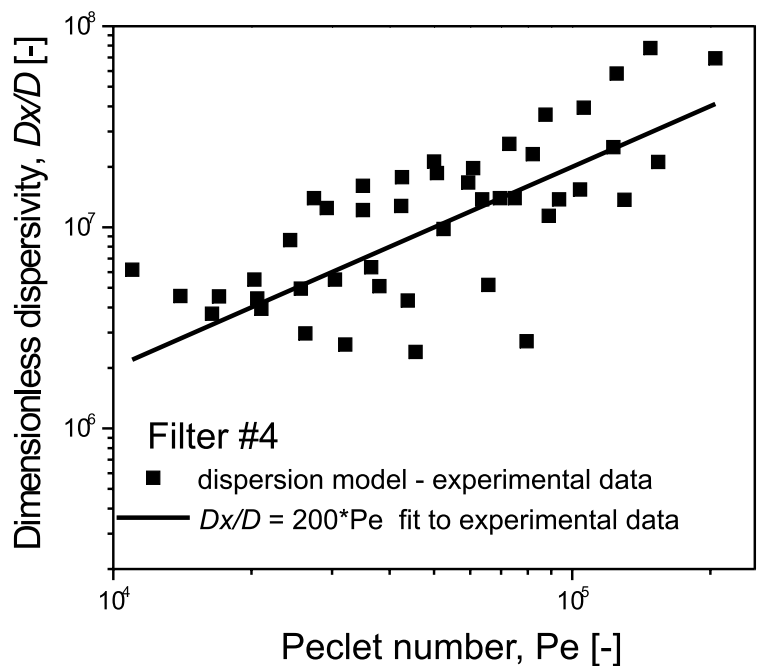

Figure 6. Dimensionless dispersivity, $D_{x} / D$, vs the Peclet number, Pe, for several air velocities

coefficient to the coefficient of Brownian diffusion was found to be nearly proportional to the Peclet number, which is in agreement with the predictions of the Koch and Brady model of convective dispersion ${ }^{7}$.

In order to find out how the phenomenon of mass dispersion in the fibrous filters affects their efficiency, for the previously determined (using the model of axial dispersion) filter coefficients, $\lambda$, the fractional penetrations were calculated according to the classical theory of depth filtration, $P=\exp (-\lambda L)$. As seen in Fig. 7, mass dispersion is an unfavourable phenomenon resulting in an increase of aerosol penetration through a filter.

\section{SUMMARY}

- The model of axial dispersion was capable of precisely describing the data obtained from the measurements for the multilayer sets of fibrous filters composed of up to nine layers of the same filter.

- For the first time ever the axial dispersion coefficients in fibrous filters were determined. It was found that the coefficients of axial dispersion, $D_{x}$, are a few orders of magnitude greater than the values of the Brownian diffusion coefficients, $D$, determined for the same particle diameters.

- The ratio of the dispersion coefficient to the coefficient of Brownian diffusion was found to be nearly proportional to the Peclet number. This suggests predominance of the convective dispersion mechanism in the highly porous fibrous filters, which have always a more or less inhomogeneous internal structure. The inhomogeneity of the fibrous filters causes a tunneling effect of the aerosol flow. It means that there are preferential flow paths through the areas of a higher local porosity.

- The results of the experiments confirmed that the mass dispersion in fibrous materials considerably reduces their efficiency.

- We can finally conclude that the mass dispersion may be a significant phenomenon in fibrous filters, especially those having a high porosity, a low thickness and a high degree of the structure inhomogeneity.

\section{SYMBOLS}

Bo - Bodenstein number

c - aerosol concentration, volume fraction

$C_{C} \quad$ - Cunningham slip correction factor

$C V$ - coefficient of variation

$d_{f} \quad$ - fiber diameter, $\mathrm{m}$

$d_{p} \quad$ - particle diameter, $\mathrm{m}$

$D \quad$ - particle diffusion coefficient, $\mathrm{m}^{2} \cdot \mathrm{s}^{-1}$

$D_{x} \quad$ - coefficient of axial dispersion, $\mathrm{m}^{2} \cdot \mathrm{s}^{-1}$

$E \quad$ - single fiber efficiency

$k_{B} \quad$ - Boltzmann constant, $\mathrm{J} \cdot \mathrm{K}^{-1}$

$L \quad$ - filter thickness, $m$

$P \quad$ - particle penetration through a filter 
a)

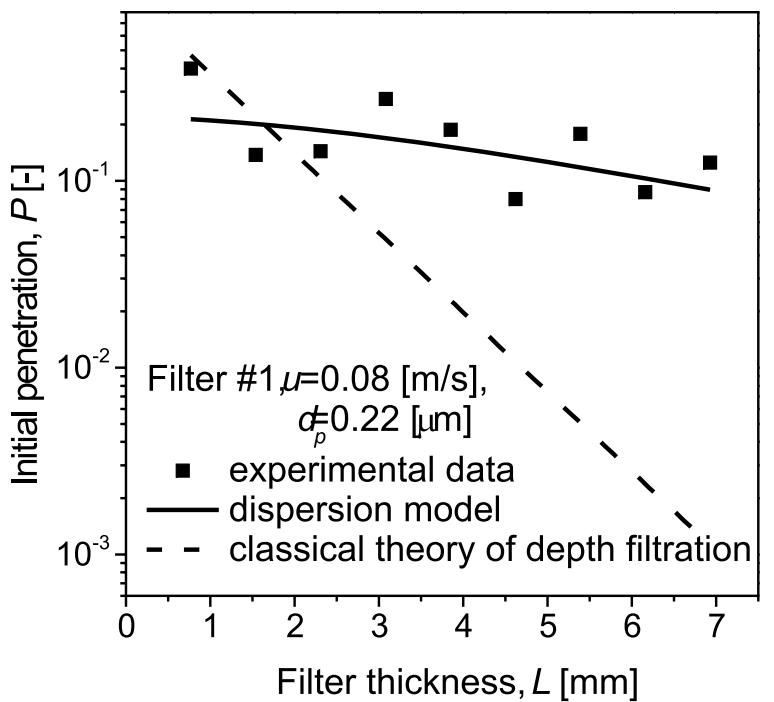

b)

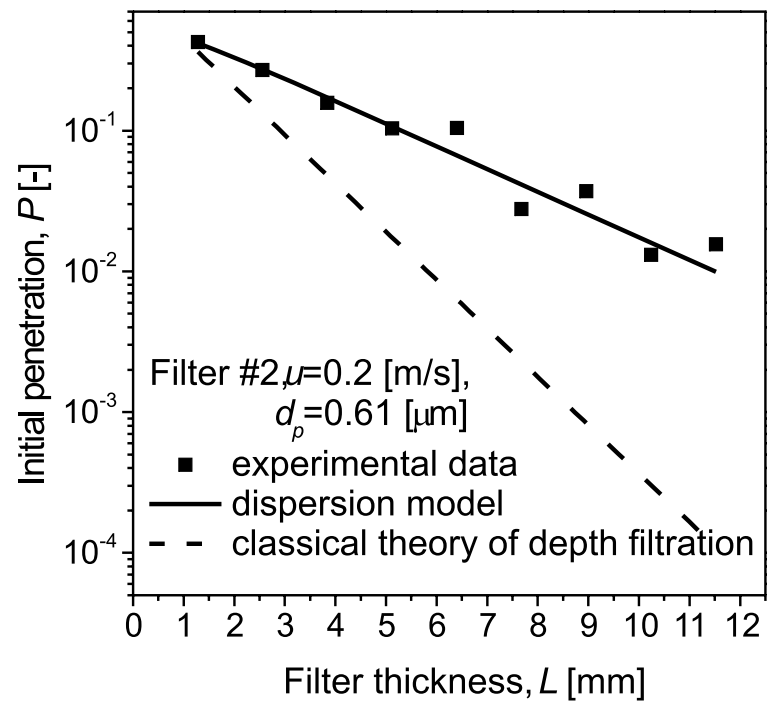

Figure 7. Comparison of the model of axial dispersion in fibrous filters used to approximate the experimental data obtained for the multilayer sets of the filter \#1 and filter \#2 with a classical theory of depth filtration predictions for the same value of $\lambda$

Pe $\quad$ - Peclet number

$q_{s} \quad$ - filter surface density, $\mathrm{g} \cdot \mathrm{m}^{-2}$

$S D \quad$ - standard deviation of a fiber diameter

$T$ - absolute temperature, $\mathrm{K}$

$u \quad$ - gas velocity, $\mathrm{m} \cdot \mathrm{s}^{-1}$

$\varepsilon \quad-$ porosity

$\eta \quad$ - filter efficiency

$\lambda \quad$ - filter coefficient, $\mathrm{m}^{-1}$

$\mu_{\mathrm{g}} \quad-$ gas viscosity, $\mathrm{Pa} \cdot \mathrm{s}$

\section{LITERATURE CITED}

1. Podgórski, A. (2002). On the Transport, Deposition and Filtration of Aerosol Particles in Fibrous Filters: Selected Problems. Warsaw, Poland: Publishing House of the Warsaw University of Technology.

2. Buyuktas, D., Wallender W.W. (2004). Dispersion in spatially periodic porous media. Heat and Mass Transfer. 40, $261-270$.

3. Lee, C.K., Sun, C. \& Mei, C. (1996). Computation of permeability and dispersivities of solute or heat in periodic porous media. Int. J. Heat Mass Transfer. 39(4), 661 - 676.

4. Koch, D.L., Cox, R.G., Brenner, H. \& Brady, J.F. (1989). The effect of order on dispersion in porous media. J. Fluid Mech. 200, 173 - 188.

5. Podgórski, A. (1999). Selected Problems in the Modelling of Aerosol Filtration in Fibrous Filters. In: Electret Filters, Production and Properties (Stenhouse, J.I.T., Gradoń, L. \& Marijnissen, J.C.M., Eds.). Delft University Press, Delft. $95-106$.

6. Bałazy, A. (2006). Aerosol Filtration in Fibrous Filters: Submicrometer and Nano-Sized Particles, Bioaerosols and Fractal-Like Aggregates. Doctoral dissertation, Warsaw University of Technology, Poland.

7. Koch, D.L. \& Brady, J.F. (1986). The Effective Diffusivity of Fibrous Media, AIChE J. 32(4), 575 - 591. 